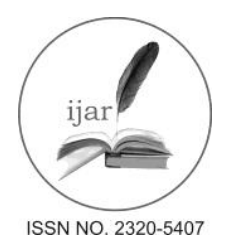

Journal homepage: http://www.journalijar.com

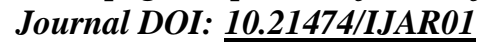

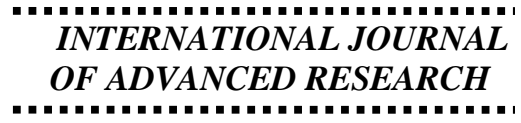

RESEARCH ARTICLE

\title{
LINOLEIC AND OLEIC ACID LEVEL IN IRAQI CHILDREN WITH CYSTIC FIBROSIS AND CELIAC
} DISEASE.

\author{
Nadia Saad Ali ${ }^{1}$, Firyal H. Al- Obaidi ${ }^{2}$, Dr. Hala Sameh Arif ${ }^{3}$. \\ 1. B.SC. College of Sciences Baghdad University \\ 2. Professor, MSC, Clinical Biochemistry, College of Medicine, AlNahrain University. \\ 3. Assistant professor, MB ChB-CABP, Consultant Pediatrician, College of Medicine, Al-Nahrain University.
}

\section{Manuscript Info}

\section{Manuscript History:}

Received: 12 April 2016

Final Accepted: 19 May 2016

Published Online: June 2016

Key words:

Cystic fibrosis, Celiac disease,

Linoleic acid, Oleic acid.

*Corresponding Author

Nadia Saad Ali.

\section{Abstract}

Introduction:- Disturbance in fatty acids metabolism and absorption occurs in patients with different causes of chronic diarrhea such as Celiac disease and Cystic fibrosis.

Objective:- measure the concentration of Linoleic acid to estimate the essential fatty acid deficiency and measure Oleic acid in Cystic fibrosis children with or without treatment, and in children with untreated Celiac disease.

Method:- This study consist of seventy children and they were : twenty children with Celiac disease, twenty children with Cystic fibrosis \{ ten of them were taking therapy (pancreatic enzyme replacement therapy and fat soluble vitamins ) and the other ten patients were not treated yet $\}$ and thirty healthy control children, they all had been taken fasting to measure Linoleic and Oleic acid

Result:- The results show that the mean \pm SD values of Linoleic acid in control group was $(0.8 \pm 0.08)$ and it was higher in $\mathrm{CF}$ patients who were taking treatment $(1.08 \pm 0.14)$, while there were a highly significant decrease in CF group who were not treated $(0.43 \pm 0.04)$ and in Celiac disease group $(0.49 \pm 0.09)$ as compared to control , in other hand the mean values of Oleic acid in control group was $(1.38 \pm 0.2)$, the mean values of Oleic acid were highly significant higher in all the study groups as compared to the control

Conclusion:- low concentration of Linoleic acid(which indicate the essential fatty acid deficiency ) with high concentration of Oleic acid were found in patients with Cystic fibrosis before they were treated and in Celiac disease patients, while the concentrations of these fatty acids were significantly high in CF patients who were taking treatment for over than three years .

Copy Right, IJAR, 2016. All rights reserved. 


\section{Introduction:-}

Celiac disease (CD) is a genetic disease and an autoimmune disorder occurring from an immune reaction to eating gluten, this reaction is occur in the small intestine, and over time it will lead to inflammation that damage the small intestine's lining which cause malabsorption and impaired digestion of some nutrients, chronic diarrhea occur in patients with $\mathrm{CD}$

For diagnosis of CD, patients should be on Gluten containing diet during the diagnostic process (NICE guideline, 2015 )

CD can be diagnosed by Anti - Gliadin anti body ( IgA , IgG ), Anti-Tissue trans glutaminase ( IgA , IgG ), EMA (anti - endomysial antibodies ) - IgA (Joseph A. Murray, et al 2000 ), the small intestine lining biopsy confirmation is the most accurate test, the biopsy is done by suction capsule or upper gastrointestinal endoscopy, the upper endoscopy is a common procedure performed by gastroenterologists (Marietta Iacucci , et al 2013)

Cystic fibrosis ( CF ) is a genetic disorder which effects mainly the lung, it's could also affect the liver, intestine and pancreas. The symptoms of CF include frequent lung infection that caused difficulty breathing, poor growth, steatorrhea, sinus infections and male infertility, weight loss, intestinal malabsorption of fat and protein, vitamin deficiency, malnutrition and the sweat gland secrete normal amount of sweat but it has high concentration of $\mathrm{NaCl}$ due to $\mathrm{Cl}^{-}$transport malfunctioning. The hepatobiliary system are damaged in about $25-30 \%$ of CF patients (Brian P O' Sullivan, et al 2009)

CF is caused by a mutation in Cystic fibrosis transmembrane conductance regulator ( CFTR ) gene which will affect on the function of the $\mathrm{Cl}^{-}$channel and lead to failure to regulate the transport of epithelial fluid in the lung, pancreas and other organs which lead to CF (Marcorelles, etal 2015) The most common mutation in CFTR gene is the deletion of phenylalanine at the amino acid position 508 ( DF508) (Jacqueline A. Jumpsen, et al 2006)

CF can be diagnosed by Sweat Chloride test which measure the amount of salt in the sweat that comes from the skin and in people older than 6 months, if the $\mathrm{Cl}^{-}$level in sweat ( $\geq 60 \mathrm{mmol} / \mathrm{L}$ ) that is an indication of $\mathrm{CF}$, or by measurement of pancreatic function ( Hara Levy, et al 2015)

Linoleic acid ( LA ) is the primary dietary omega - 6 poly unsaturated fatty acid ( PUFA ), it's an 18 - carbon fatty acid with 2 double bonds ( 18:2 omega-6), LA is an essential fatty acid because its cannot be synthesized in human bodies ( William S . Harris, at al 2009)

Oleic acid is a mono unsaturated fatty acid ( MUFA ) or Omega -9 that is found in animal and vegetable oil, its chemical formula $\mathrm{C}_{18} \mathrm{H}_{34} \mathrm{O}_{2}$ it's found in high amount in olive oil , and sunflower oil. ( David Tin Win , 2005)

In untreated CD, fat malabsorption can occur and the concentration of MUFA are higher in CD patients when compare it with healthy persons (Solakivi T, et al 2009). Most patients with CD were found to have decreased the essential fatty acids concentration (Krums LM , 2011)

Patients with CF have altered unsaturated fatty acid composition, especially in serum or whole blood, and the most common is the decrease in LA and docosahexaenoate with increase in Palmitoleate, Oleic, Arachidonic acid ( AA ) and eicosapentaenoate ( Adam C. Seegmiller , 2014)

So essential FA estimation has been found to be useful in follow up of patients treatment for these diseases and to guide the response to therapy.

\section{Materials and methods:-}

The research was a case control study. It was conducted in Al Imamain Al - Kadhimen medical city (Pediatric department ), Gastroenterology and Hepatology Center ( Medical City ) and Central Public Health Laboratory . The study protocol was approved by the Ethical Committee of College of medicine $\backslash$ AL- Nahrain University .

Seventy children were included in this study during the period from mid of November 2015 to April 2016 . Information was taken by a questionnaire paper after taking an informed consent from their parents, the 
questionnaire paper include patient name, age, education of parents, kinship between parents, clinical feature , clinical exam ( as taken from the patient's medical records ), blood or its product transfusion, bottle or breast feeding, fasting, drug, stool characteristic , frequency, looseness , quantity and color , time of diagnosis.

The patients age was between ( 2 - 18 ) years and they were ( 20 ) children with CD, ( 20 ) children with CF, and ( 30 ) control children

All of these children were fasting and all of them except the control were born from relative parents who were not educated or only finishing primary school. All patients were examined clinically and diagnosed by pediatrician.

Celiac disease:- their age was between ( 2-11) years, they were diagnosed by Anti - Gliadin anti body ( $\operatorname{IgA}, \operatorname{IgG}$ ) , Anti - Tissue trans glutaminase ( IgA , IgG ) in Al- Imamain Al- Kadhimen medical city and in Central Public Health Laboratory ( Immunity department ) which were positive, and by duodenal biopsy at the Gastroenterology and Hepatology center. All the patients who had been taken were not treated yet by a Gluten free diet and they had either chronic or recurrent diarrhea.

Cystic fibrosis:- patients were diagnosed by Sweat Chloride test which was greater than or equal to $60 \mathrm{mmol} / \mathrm{L}$ and they were :

* Ten patients were taking Pancreatic enzyme replacement therapy, fat soluble Vitamin ( A, K, D, E ) for over than three years and their age was between $(5-17)$ years.

* Another ten patients with CF , their age was between ( $2-6)$ years, they were taken not treated yet and they had steatorrhea .

Control children:- they either admitted to the hospital without having any of these diseases or without having any of the GIT disorder, or coming to the hospital for routinely check up , and their age was between ( $2-17$ ) years

Exclusion criteria:- Children below two years, children with tonics, children with bottle feeding, children who had recent transfusion of blood or its products within the last month, children taking drugs that effects lipids such as ( Cholestyramin )

\section{Blood Sample Collection:-}

About $2.5 \mathrm{ml}$ of venous blood samples was taken and added to the EDTA tube and then one drop of blood was add to each one of the 8 circles on the tandem mass special filter paper by using pasture pipette, and then left to dry for about ( 30 ) minutes at room temperature, the blood spots were not touched to avoid contamination and kept away from direct sunlight and humidity, after that it was sent to laboratory of Saint Joseph hospital / Lebanon / Beirut for measuring ( Linoleic and Oleic ) by using tandem mass spectrometry ( MS /MS technology ) .

\section{Statistical analysis:-}

Data were analyzed by statistical packages of SPSS 20 ( statistical packages for social sciences - version 20 ) and Microsoft excel 2013 . All data were presented as a mean \pm Sd . Statistical comparisons of continuous variables among each one of the groups were performed by independent $t-$ test . For all tests $p-$ value $<0.05$ was considered statistically significant and $<0.001$ was considered highly significant.

\section{Results:-}

The current study included seventy children and they were ( 20 ) children with untreated Celiac disease , ( 20 ) children with Cystic fibrosis ( 10 ) of them were taken treatment for over than three years and the other ( 10 ) children were not treated yet, while the control group in this study were 30 children

Table ( 1 ) revealed that the mean values of Linoleic acid was significantly higher in CF patients who were taking treatment as compared to control, while there were a highly significant reduction in CF group who were not treated and in untreated celiac disease group as compared to control $(\mathrm{p}<0.001)$

In other hand the mean values of Oleic acid were significantly higher in all the study groups as compared to the control $(\mathrm{p}<0.001)($ table 1$)$ 
Table 1:- Comparison of Linoleic and Oleic between control and each of the three patients groups by independent $t$ test

\begin{tabular}{|l|l|l|l|l|}
\hline Parameters & $\begin{array}{l}\text { Control } \\
(\mathbf{n = 3 0}) \\
\text { mean } \pm \text { SD }\end{array}$ & $\begin{array}{l}\text { CF on treatment } \\
(\mathbf{n = 1 0}) \\
\text { mean } \pm \text { SD }\end{array}$ & $\begin{array}{l}\text { Untreated CF } \\
(\mathbf{n = 1 0}) \\
\text { mean } \pm \text { SD }\end{array}$ & $\begin{array}{l}\text { Celiac disease } \\
(\mathbf{n = 2 0}) \\
\text { mean } \pm \text { SD }\end{array}$ \\
\hline Linoleic $(\mu \mathrm{M})$ & $0.8 \pm 0.08$ & $1.08 \pm 0.14^{* *}$ & $0.43 \pm 0.04^{* *}$ & $0.49 \pm 0.09^{* *}$ \\
\hline Oleic $(\mu \mathrm{M})$ & $1.38 \pm 0.2$ & $2.9 \pm 0.48^{* *}$ & $2.7 \pm 0.51^{* *}$ & $1.88 \pm 0.21^{* *}$ \\
\hline
\end{tabular}

* Comparison with control $\mathrm{P}$ value $<0.05$, ** Comparison with control $\mathrm{P}$ value $<0.001$

A comparison of Linoleic and Oleic between all the study groups shows that the mean value of LA was higher in CF group who were taking treatment and lower in $\mathrm{CF}$ without treatment as compared to the other study groups.

While Oleic acid was higher in all the study groups as they compared to the control, its level was much higher in $\mathrm{CF}$ with and without treatment groups than its level in $\mathrm{CD}$ and control groups as seen in figure (1)

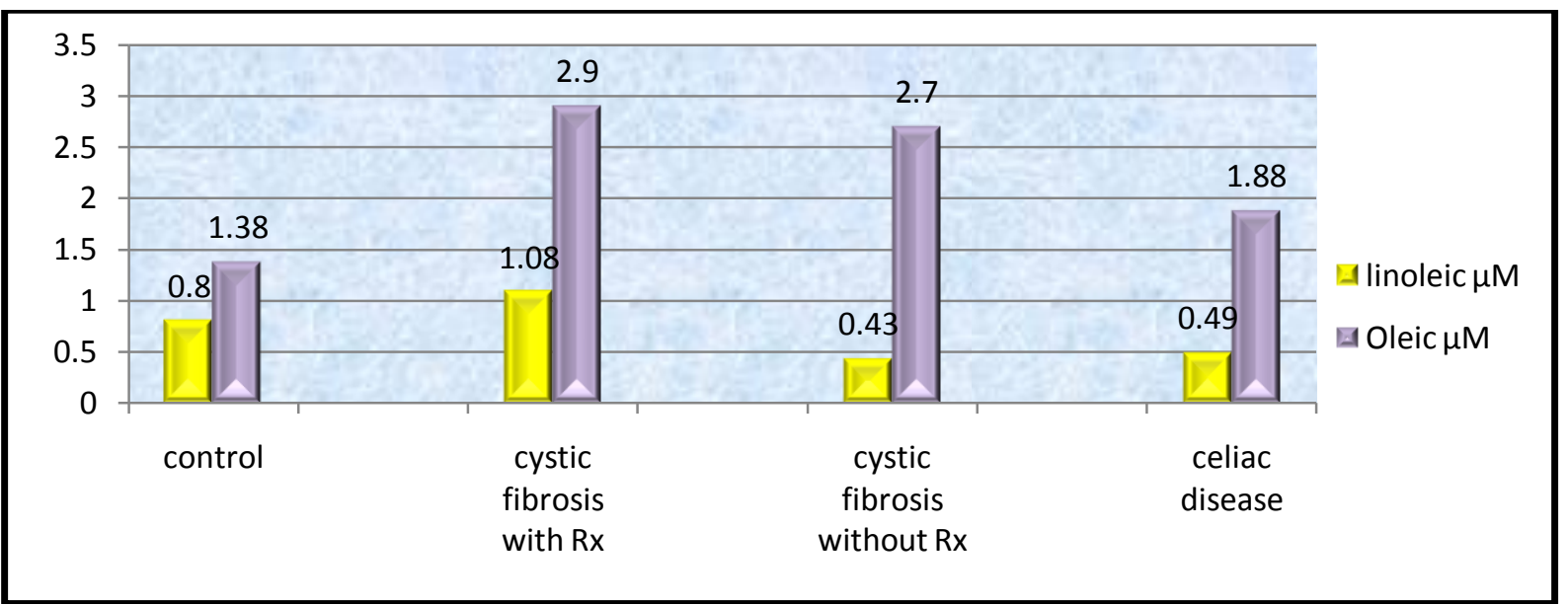

Figure 1: Comparison of Linoleic and Oleic in different study groups.

\section{Discussion:-}

Malabsorption and malnutrition occur in children with different causes of chronic diarrhea, in this study children with $\mathrm{CF}$ and $\mathrm{CD}$ were studied.

Children with chronic diarrhea had fatty acids disturbance, essential fatty acid deficiency ( especially Linoleic acid )

In this study fatty acid ( LA, Oleic acid ) were significantly increased in CF patients after over than three years of treatment with PERT and fat soluble vitamins ( A, K, D , E ) as seen in table (1)

Some patients with CF succeed in recovering from malnutrition and growth faltering after treatment initiation while others fail to succeed ,so sustained high energy intake and normal plasma essential fatty acid status are critical determinants for treatment responsiveness within 2 years after diagnosis of CF.( Shoff SM, et al 2006)

Sustained normal linoleic acid ( $\uparrow$ LA ) and high energy intake ( $\uparrow \mathrm{EN})$ status are critical determinants of treatment responsiveness within 2 years after CF diagnosis, they are important in promoting children's adequate weight gain with newly diagnosed CF. This suggests the need for frequent monitoring of plasma LA in patients with newly diagnosed CF and in all subjects with CF. Decreased LA status was observed in patients with less effective PERT, more severe lung disease, and worse nutritional status ( Walkowiak, et al 2007)

Untreated CF patients show a highly significant decrease in LA, with highly significant increase in Oleic acid ( table 1 ), lower levels of LA are due to increased its metabolism to AA via $\Delta-6$ desaturase enzyme (Neeraj Vij , 2010)

Increase the level of Palmitoleate, Oleate with changes in the omega $-3 \mathrm{~s}$ and omega $-6 \mathrm{~s}$ is correlated with increased the expression of FA metabolic enzymes. CF cells in culture show increased metabolism along the metabolic 
pathways which lead to $18: 1 n-9,20: 3 n-9,16: 1 n-7$, these changes are accompanied by increased expression of the enzymes which produce these FA. These findings suggest that FA abnormalities of the n-7 and n-9 series are result, at least in part, of increased expression and activity of these metabolic enzymes in CFTR - mutated cells . (Thomsen $\mathrm{KF}$, et al 2011) the gold standard for observing PERT is fat balance . (Mini Kalivianakis, et al 1999)

Children with CD had a significant decrease in LA with increase in the mean value of Oleic ( table1)

In CD intestinal villi are flattened and shortened, the epithelial cells damaged and increased the depth of crypt layer this all will compromise the intestinal absorption ( Murray JA , 1999)

Patients with CD have a high requirement for repairing the intestinal tissue and there is an increase of the conversion of EFA into eicosanoids, so this together with compromised intestinal absorption will lead to the patient with CD is predicted to be at a high risk for having essential fatty acid deficiency ( Branski D, et al 1992)

Patients with active CD had increased Palmitoleic ( 16:1n-7), Oleic (18:1n-9) and Mead acid (20:3n-9) (Steel , Daniella M. , et al 2006)

In a comparison of FAs between all the study groups ( figure1 ), the mean value of LA was higher in CF group who were taking treatment while it was lower in CF without treatment . Otherwise Oleic acid was higher in all the study groups as they compared to the control, and it was much higher in CF groups whether they were treated or not

List of abbreviation

\begin{tabular}{|l|l|}
\hline CD & Celiac disease \\
\hline CF & Cystic fibrosis \\
\hline CFTR & Cystic fibrosis transmembrane conductance regulator \\
\hline LA & Linoleic acid \\
\hline FA & Fatty acid \\
\hline
\end{tabular}

\section{Acknowledgements:-}

Grateful thanks and resections to Prof . Firyal H . Al - Obaidi , and I would like to express my deep respect to Dr . Hala sameh arif for her great help, kindness, guidance and encouragement.

I would like to express my grateful thanks to all my seniors in Department of Chemistry and Biochemistry in $\mathrm{Al}-$ Nahrain University / College of Medicine.

\section{Reference:-}

1. Adam C. Seegmiller ( 2014 ): Abnormal unsaturated fatty acid metabolism in cystic fibrosis : biochemical mechanisms and clinical implications . Int.J.Mol.Sci. ,15(9):16083-16099

2. Branski D, Hurvitz H, Halevi A, et al (1992) : Eicosanoids content in small intestinal mucosa of children with celiac disease. J Pediatr Gastroenterol Nutr ,14:173-6

3. Brian P O' Sullivan, Steven D Freedman ( 2009 ) : Cystic fibrosis . Lancet ,373 (9678): 1891-904.

4. David Tin Win ( 2005 ) : Oleic Acid - The Anti - Breast Cancer Component in Olive Oil . AU J.T. , 9(2): 75-78

5. Di Sabatino A, Corazza GR ( 2009 ): Coeliac disease . Lancet , 373 (9673):1480-93.

6. Hara Levy , Philip M . Farrell (2015 ) : New Challenges in the Diagnosis and Management of Cystic Fibrosis . The journal of pediatrics , 166(6):1337-1341

7. Jacqueline A . Jumpsen a , Neil E . Brown, Alan B.R. Thomson, S.F. Paul Man, Yeow K. Goh, David Ma c , et al (2006): Fatty acids in blood and intestine following docosahexaenoic acid supplementation in adults with cystic fibrosis . Journal of Cystic Fibrosis , $5: 77-84$

8. Joseph A. Murray, Judith Herlein , Frank Mitros , James A. Goeken ( 2000 ) : Serologic Testing for Celiac Disease in the United States : Results of a Multilaboratory Comparison Study . Clincal and diagnostic laboratory immunology , 7(4): 584-587.

9. Krums LM ( 2011 ) : Deficit of polyunsaturated fatty acids in patient with celiac disease . Eksp Klin Gastroenterol , (30):31-4

10. Marcorelles P, Gillet D, Friocourt G, Ledé F, Samaison L, Huguen G, Ferec C ( 2012 ) : Cystic fibrosis transmembrane conductance regulator protein expression in the male excretory duct system during development . Hum . Pathol , 43 (3): 390-7. 
11. Marietta Iacucci, Subrata Ghosh ( 2013 ) : Routine duodenal biopsies to diagnose celiac disease . Can J Gastroenterol , 27(7): 385.

12. Mini Kalivianakis, Deanna M Minich, Charles MA Bijleveld, Wim MC van Aalderen, Frans Stellaard, Marianne Laseur, et al. ( 1999 ) : Fat malabsorption in cystic fibrosis patients receiving enzyme replacement therapy is due to impaired intestinal uptake of long - chain fatty acids . American Society for Clinical Nutrition , 69 ( 1) :127-134

13. Murray JA , Rubio - Tapia A ( 2012 ) : Diarrhoea due to small bowel diseases . Best Pract Res Clin Gastroenterol , 26 (5):581-600

14. National institute for health and care excellence ( NICE) guideline. Coeliac disease : recognition , assessment and management 2 September 2015

15. Neeraj Vij ( 2010 ) : Linoleic acid supplement in cystic fibrosis : friend or foe ? American journal of physiology lung cellular and molecular physiology , 299(5) : L597-L598.

16. Shoff SM, Ahn HY, Davis L, Lai H ; Wisconsin CF Neonatal Screening Group ( 2006) : Temporal associations among energy intake, plasma linoleic acid, and growth improvement in response to treatment initiation after diagnosis of cystic fibrosis. Pediatrics ,117(2):391-400.

17. Solakivi T, Kaukinen K, Kunnas T, Lehtimäki T, Mäki M, Nikkari ST ( 2011 ) : Serum fatty acid profile in subjects with irritable bowel syndrome . Scand J Gastroenterol , 46 (3):299-303

18. Steel, Daniella M .; Ryd, Walter; Ascher, Henry; Strandvik, Birgitta. ( 2006 ) : Abnormal Fatty Acid Pattern in Intestinal Mucosa of Children With Celiac Disease Is Not Reflected in serum phospholipid . Journal of Pediatric Gastroenterology \& Nutrition, 43(3): pp 318-323

19. Thomsen KF, Laposata M, Njoroge SW, Umunakwe OC, Katrangi W, Seegmiller AC ( 2011 ) : Increased elongase 6 and $\Delta 9$-desaturase activity are associated with $n-7$ and n-9 fatty acid changes in cystic fibrosis . Lipids , 46(8):669-77

20. Walkowiak , Jaroslaw ; Lisowska , Aleksandra ; Blaszczynski, Michal; Przyslawski, et al ( 2007 ) : Polyunsaturated Fatty Acids in Cystic Fibrosis Are Related to Nutrition and Clinical expression of the Disease . Journal of Pediatric Gastroenterology \& Nutrition , 45 (4 ): p488-489

21. William S . Harris, Dariush Mozaffarian, Eric rim, Penny Kris-Etherton, Lawrence L. Rudel, Lawrence J.Appel, et al ( 2009 ) : Omega-6 Fatty acids and risk Risk for Cardiovascular Disease. Circulation , 119:902907 